\title{
Molecular Dissection of Lymphocyte Signal Transduction Pathways
}

\author{
ROGER M. PERLMUTTER \\ Department of Immunology, Howard Hughes Medical Institute, University of Washington School of Medicine, \\ Seattle, Washington 98195
}

\begin{abstract}
The earliest biochemical changes in Iymphocyte activation include the accumulation of phosphotyrosine-containing proteins. This accumulation, catalyzed by specific protein tyrosine kinases, is required for subsequent cell activation. Experimental manipulation of these protein tyrosine kinases results in improved or decreased transit through the activation sequence. Analysis of transgenic animals bearing altered kinase genes permits assembly of a model for signal transduction from the $T$-cell antigen receptor. (Pediatr Res 33 (Suppl): S9-S15, 1993)
\end{abstract}

Shortly after crosslinking of the T-cell antigen receptor by cognate ligand, a series of biochemical changes results. The earliest observable events are changes in the accumulation of phosphorylated substrates, particularly a set of proteins specifically phosphorylated on tyrosine residues. This accumulation of substrates begins within seconds after ligand occupancy of the T-cell antigen receptor. Thereafter, changes in the turnover of membrane phospholipids (Fig. 1) and the accumulation of high concentrations of intracellular calcium occur transiently. All these alterations precede changes in gene expression, especially the activation of a set of genes important for proliferation. Included among these are genes encoding the transcription factors, genes encoding lymphokine receptors, and the lymphokine genes themselves (1). These events together permit lymphocytes to proceed along a course that ultimately will lead to DNA replication and proliferation.

Two fundamental problems exist in understanding this sequence of events. First, we have no idea of the causal relationships among these various biochemical alterations. Is tyrosine phosphorylation a cause of these subsequent events? Second, we have no idea how the receptor molecules on the surfaces of lymphoid cells effect these biochemical changes. This article focuses on that problem.

Arrayed on the $T$ lymphocyte surface is a set of molecules that transmit signals to the cell interior. These structurally related molecules are all encoded by genes that are members of the Ig superfamily. They include the T-cell antigen receptor $\alpha$ and $\beta$ chains (2), the associated CD3 components of the T-cell receptor signaling complex (3), the coreceptors CD4 and CD8 (4), and other auxiliary signaling structures. All these molecules also share another aspect of structure: none has any obvious means of transmitting signals to the cell interior in that they all have relatively short cytoplasmic extensions that lack known catalytic function.

Some years ago, my group and others began studying the various signal transduction mechanisms that might be responsi-

Correspondence: Roger M. Perlmutter, M.D, Ph.D., Department of Immunology, University of Washington School of Medicine, Health Sciences Building, Mail Stop SL-05, Seattle, WA 98195.

Supported in part by a grant from the NIH (CA045682) and by the Howard Hughes Medical Institute. ble for coupling these receptors to the cell interior. We were particularly intrigued by the idea that lymphocyte-specific protein tyrosine kinases might couple these receptors to other biochemical pathways. Our theory was not derived from detailed knowledge about the importance of tyrosine phosphorylation. It simply seemed that in many respects the signal transduction cascade initiated by lymphocyte cell surface receptors resembled the kind of signal transduction processes occurring with stimulation of growth factor receptors known to possess intrinsic protein tyrosine kinase activity (5).

With this hypothesis in mind, we studied a variety of lymphoid cell lines, searching for those with elevated tyrosine kinase activity. One of the cell lines-a mouse Moloney murine leukemia virus-transformed cell line called LSTRA-was particularly informative.

The LSTRA cell line was found to contain high levels of a specialized protein tyrosine kinase of $56 \mathrm{kD}$ apparent molecular mass. We were able to use molecular biologic techniques to identify the gene that encodes this molecule (6) and learned that it is a typical nonreceptor protein tyrosine kinase of the type exemplified by $\mathrm{p} 60^{\text {src }}$.

The $s r c$-family of protein tyrosine kinases all share a similar structure. These molecules are myristoylated at their amino termini, which presumably assists their interaction with the inner leaflet of the plasma membrane. Across the entire structure, three distinct regions can be identified (Fig. 2). First, a unique amino terminal domain structure, about 70 amino acids in length, appears to control direct interaction with receptor structures. Then a set of $s r c$ homology motifs appears, including sequences that are involved in the binding of phosphotyrosine itself, and, finally, there is a carboxyterminal kinase domain, the business end of the molecule responsible for phosphorylating substrates (7). Included in the kinase domain is a critical site of regulation: a tyrosine near the carboxy terminus that is ordinarily phosphorylated in vivo (8). If the phosphate is stripped from this site, the kinase becomes much more active. Study of this activity can be useful in investigating the function of these molecules.

There are now eight well-described members of the $s r c$ family. It is important to remember that these molecules are usually specialized for expression in hematopoietic cells. For example, the $b l k$ kinase identified by Steve Desiderio and his colleagues at Johns Hopkins University (9) is expressed only in B lymphocytes. It performs some unique signal transduction function in those cells. The $h c k$-encoded kinase is expressed primarily in granulocytes (10). The $l c k$ gene, which we originally cloned from LSTRA cells, is expressed only in lymphoid cells, particularly $T$ lymphocytes (6). Thus, the $l c k$ gene is an ideal candidate for a signal transduction molecule that might link lymphoid cell surface receptors to the cell interior.

But what precise function does this protein tyrosine kinase perform? Lck transcripts accumulate to especially high levels in thymocytes. This suggests that the $l c k$ gene performs some function critical to $\mathrm{T}$ lymphocyte development. Moreover, the lck gene is expressed at the earliest time that one can identify T-cell 


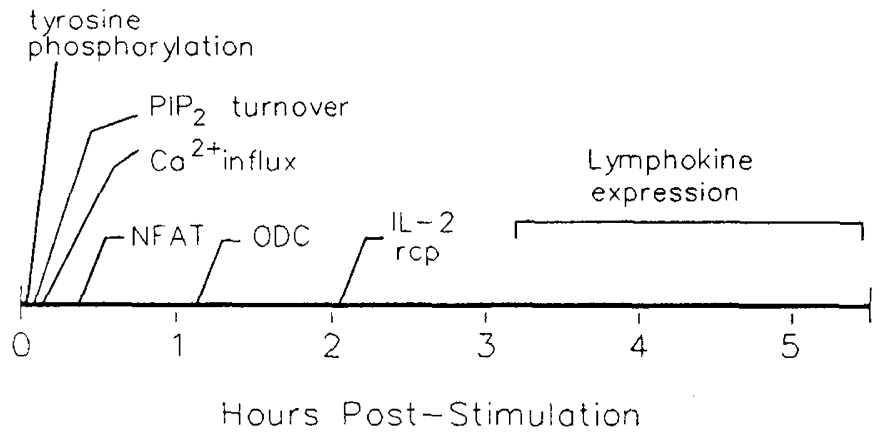

Fig. 1. Early metabolic events after T-cell activation. Shown is a hypothetical time line for activation listing some of the major early biochemical changes that occur after T-cell receptor stimulation. NFAT is a transcription factor complex involved in regulating IL-2 expression. $O D C$ is ornithine decarboxylase. $P I P_{2}$ is phosphatidylinositol-bis-phosphate. $I L-2 r c p$ is interleukin-2-receptor.

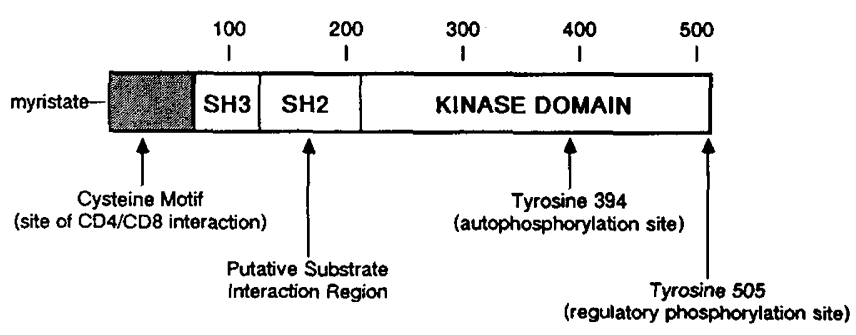

Fig. 2. Structural concept of a typical $s r c$-family protein tyrosine kinase. Shown is a schematic representation of $p 56^{\text {lck }}$ illustrating the positions of the amino-terminal and "src-homology" $(\mathrm{SH} 3, \mathrm{SH} 2)$ domains. See text for details.

precursors in the thymus and throughout the set of maturation stages defining $\mathrm{T}$-cell development.

The structure of $\mathrm{p} 56^{\text {lck }}$ closely resembles that of a number of oncoproteins, suggesting that it may be involved in controlling replication. Indeed, it is possible to demonstrate directly that the lck gene is an oncogene that can confer on certain cells the ability to grow in the absence of appropriate growth factors and appropriate substrate contacts. For example, we have used transcriptional regulatory sequences from the mouse metallothionein promoter to drive the expression of normal and mutated lck cDNA. Mutations were introduced at the $3^{\prime}$ end that change a regulatory tyrosine codon to a phenylalanine or to a termination codon.

Wild-type fibroblasts transfected with constructs that direct the expression of the ordinary lck gene do not exhibit morphologic abnormalities. However, the mutated forms of the $l c k$ gene efficiently and successfully transform these fibroblasts, yielding cells that become highly refractile, elaborate long spindly processes, and adhere poorly to substrates. These $l c k$ fibroblasts grow in a contact-independent fashion, form tumors in nude mice, form colonies in soft agar, and, by all criteria, are transformed. Thus, a single-point mutation unmasks the transforming potential of the lck gene (11).

Intriguingly, the lck gene is positioned at a site (1p34) of frequent chromosomal abnormalities in human malignant disease, particularly non-Hodgkin lymphomas (12). Recently, two groups have demonstrated that the lck gene is positioned at the breakpoints of certain chromosomal translocations observed in human T cell acute lymphoblastic leukemias (13). In these cases, the $l c k$ gene was juxtaposed with the T-cell receptor $\beta$ locus on chromosome 7 . These observations provide reason to believe that the $l c k$ gene can contribute to the pathogenesis of at least some forms of human malignant disease, particularly lymphoid malignancies.
None of this theorizing advances our understanding of what the lck gene actually does; however, it suggests that $\mathrm{p} 56^{\text {lck }}$ may behave as a signal transduction molecule involved in the control of replication or those early activation events in $\mathrm{T}$ lymphocytes that give rise to proliferation. Recent studies demonstrate that p56 $6^{\text {lck }}$ physically associates with the CD4/CD8 coreceptor molecules on the T-cell surface. These observations argue that $\mathrm{p} 56^{\text {lck }}$ may be brought into the $\mathrm{T}$-cell receptor complex at the time of antigen recognition and may be directly involved in transmitting signals from that antigen receptor complex.

Studies by our group and others have defined the mechanism of association of $\mathrm{CD} 4$ and $\mathrm{CD} 8$ with $\mathrm{p} 56^{\text {lck }}$ and have shown that cysteine residues near the $\mathrm{N}$ terminus of $\mathrm{p} 56^{\text {lck }}$ and the $\mathrm{C}$ terminus of these coreceptors are crucial for this interaction. Interruption of any of these four cysteine residues completely destroys the association (14).

Thus, the amino terminal domain of $\mathrm{p} 56^{\text {lck }}$ is involved in permitting the molecule to associate with the $\mathrm{T}$-cell antigen receptor complex by its interaction with the coreceptors, CD4 and CD8. This observation gives rise to a fairly simple hypothesis: Under ordinary circumstances, $\mathrm{p} 56^{\text {lck }}$ is in some sort of equilibrium state interacting with this coreceptor. At the time of antigen recognition, $\mathrm{p} 56^{\text {lck }}$ becomes involved in the complex and becomes activated, presumably by dephosphorylation of a carboxy terminal tyrosine residue. Indeed, several groups have demonstrated that the CD45 phosphotyrosine phosphatase can dephosphorylate tyrosine 505 and hence activate $\mathrm{p} 56^{\text {lck }}$ in precisely the fashion previously explored using mutant $l c k$ cDNA introduced into fibroblasts (15).

To test this model, we hoped to artificially augment or decrease the amount of $p 56^{\text {lck }}$ activity present in otherwise normal $T$ lymphocytes, that is, in transgenic mice. Such experiments have numerous advantages over conventional transfection studies. First, the signaling molecules are expressed in a normal cellular context, obviating the use of cell lines that invariably exhibit abnormal signaling properties. Second, all potentially relevant lymphocyte subsets can be simultaneously examined. It is not clear that all lymphocyte subsets can be cultured in vitro with the same efficiency. The advantage of using this in vivo system is that all potentially relevant lymphocyte subsets can be simultaneously targeted. Finally, the significance of signaling pathways in lymphocyte development is readily addressed in transgenic mice, and there are no satisfactory in vitro models for lymphocyte development.

To perform these experiments, it was necessary to characterize transcriptional regulatory elements that would permit us to direct the expression of signaling molecules, specifically to lymphocyte subsets. The transcriptional regulatory element that we selected is derived from the $l c k$ gene itself. The $l c k$ gene has two transcriptional regulatory regions separated by 35000 bases of germline DNA. These two "promoters," the proximal or 3' promoter and the distal or $5^{\prime}$ promoter, function in a developmentally regulated fashion $(16,17)$. The proximal promoter is active principally in thymocytes and turns off at the time that thymocytes exit the thymus and populate the periphery. In contrast, the distal promoter is somewhat active in the thymus, but functions primarily in peripheral $\mathrm{T}$ ceils. Through careful examination of the regions adjacent to these transcription start sites, we identified sequence elements capable of directing the tissue-specific and temporally correct expression of heterologous DNA elements in transgenic animals.

We have used these promoter elements to manipulate the expression of three potentially important signaling molecules: guanine nucleotide-binding proteins of the $\mathrm{Gi}$ type, $\mathrm{p} 56^{\text {lck }}$ itself, and another closely related protein-tyrosine kinase-p $59^{\text {fyn }}$. Each set of experiments has proved quite revealing.

To examine the function of $\mathrm{p} 56^{\text {lck }}$, a piece of the lck gene, including all of its coding region and the proximal $l c k$ promoter, was juxtaposed with $3^{\prime}$ untranslated region sequences derived from the hGH gene. These hGH sequences were included to 
provide a reasonable, reliable marker for expression at the RNA level.

Using an $\mathrm{hGH}$ probe, expression of the transgene is observed in the thymus, but not in the liver, kidney, heart, or spleen. These are the expected results given that $l c k$ promoter is active in the thymus but turns off at the time that thymocytes migrate to the periphery.

The consequences of this overexpression of $\mathrm{p} 56^{\text {lck }}$ are dramatic. Animals expressing the wild-type lck transgene at levels that are 4- to 5-fold above normal (Fig. 3) all develop large thymic tumors (18). The tumors are usually detectable by $3 \mathrm{wk}$ of life, and most of the animals are dead by the time they are 2 mo old, which presents difficulties when one wishes to maintain lines of these mice. The tumors invariably consist of immature thymoblasts that do not have T-cell receptors on their surfaces, and in most cases these thymoblasts are heterogeneous with respect to expression of CD4 or CD8.

The pattern of tyrosine phosphorylation in these cells is remarkable in its close resemblance to that observed in the LSTRA tumor cell line that we originally used to define the $l c k$ gene. Simply looking at the pattern of phosphotyrosine accumulation using a phosphotyrosine-immunoblotting technique in LSTRA (Fig. 4) reveals an enormous number of proteins phosphorylated on tyrosine reflecting the 50 -fold overexpression of $\mathrm{p} 56^{\text {lck }}$ in the cells. Thymocytes from a normal mouse contain relatively few proteins phosphorylated on tyrosine. In contrast, thymocytes from animals bearing the lck transgene exhibit very high levels of phosphotyrosine-containing proteins. The pattern of substrates in these thymocytes closely resembles the pattern in the LSTRA cell line. Hence, by generating these transgenic animals, we have essentially duplicated what occurred in LSTRA cells, where retroviral promoter insertion activates the lck gene. We have also, in part, provided a model for the human disease that results from translocation of this gene. Unfortunately, this model does not tell us much about the signal transduction function of this gene, except that when activity is at high levels it can certainly promote the growth of immature thymoblasts.

In subsequent experiments, carried out in collaboration with Tada Tanaguchi and his colleagues in Osaka, we have shown that $\mathrm{p} 56^{\text {lck }}$ interacts with another cell surface receptor, the receptor for IL-2 (19). The IL receptor consists of three chains (20). The $\beta$ chain seems to provide the bulk of the signaling function.

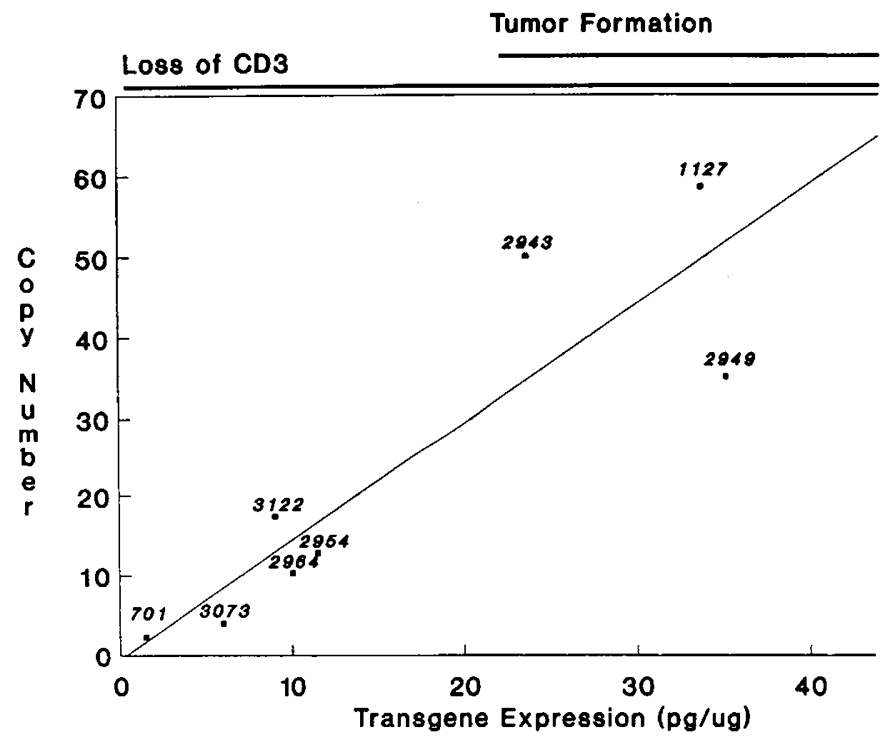

Fig. 3. High-level expression of p56 $6^{\text {lck }}$ provokes thymoma develop ment. Copy number-dependent expression of an $l c k$ transgene is observed in these eight transgenic mouse strains. Only those strains expressing very high levels of the transgene mRNA develop thymic tumors. See reference 18 , from which this figure was adapted, for details.

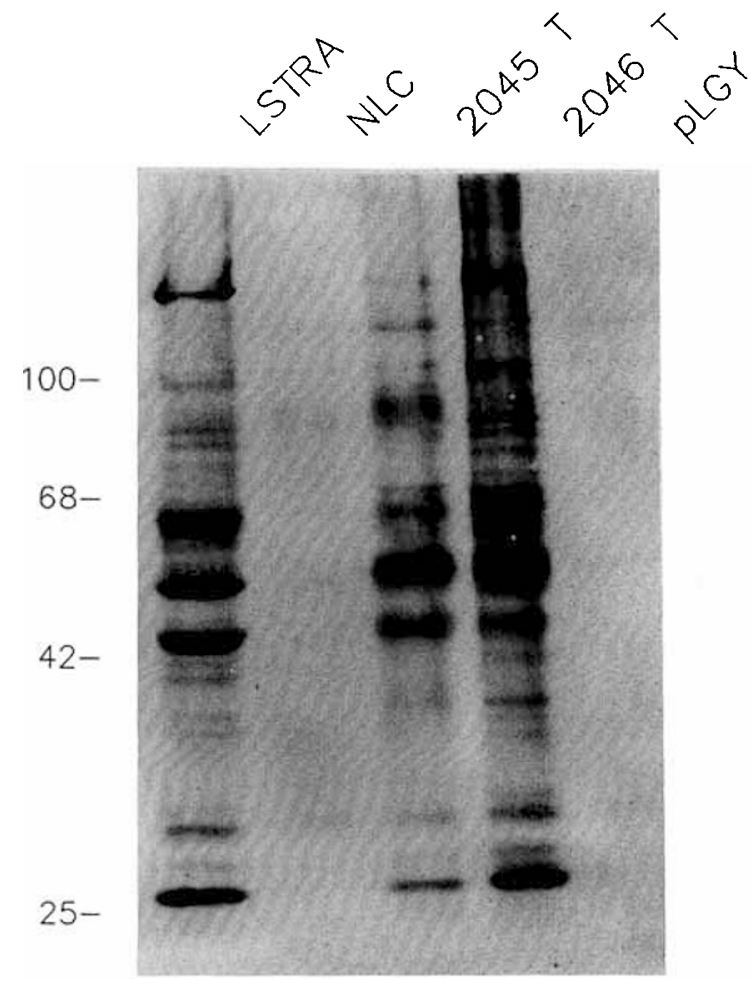

Fig. 4. Accumulation of phosphotyrosine-containing substrates in cells expressing high levels of p56 lck. The lck gene is rearranged and overexpressed in the LSTRA cell line as a result of a retroviral promoter insertion event. Thymoma cells from lck transgenic mice (2045 T, 2046 $T)$ contain similarly high levels of phosphotyrosine-containing proteins as compared with thymocytes from normal littermate controls $(N L C)$ or from animals expressing lower levels of a wild-type transgene ( $p L G Y)$. Adapted from reference 18 .

It binds IL-2 with intermediate affinity by itself and interacts with the kinase domain of $\mathrm{p} 56^{\text {lck. }}$. If one adds IL-2 to normal cells, p56 $6^{\text {lck }}$ activity increases. Hence, at least two cell surface structures can interact with $\mathrm{p} 56^{\text {lck }}$ to give rise to signal transduction events. In at least some cases, stimulation of p56 clearly contributes to the control of T-cell mitogenesis. But p56 ${ }^{\text {lck }}$ is not the only nonreceptor protein tyrosine kinase that functions in $\mathrm{T}$ cells. Our experiments have also demonstrated a fundamental role for a second $s r c$-family kinase, $\mathrm{p} 59^{f y n}$. The fyn gene is transcribed at high levels in both neuronal cells and $\mathrm{T}$ lymphocytes (21). Moreover, there exist two alternative forms of p5 $9^{f y n}$, products of the same gene, that differ as a result of mutually exclusive alternative splicing of two distinct exons 7 . One, exon $7 \mathrm{~B}$, is incorporated into fyn transcripts that are expressed in lymphocytes. The other, exon $7 \mathrm{~A}$, contributes to fyn transcripts in the brain $(21,22)$.

Remarkably, p59 $9^{f y}$ is first expressed at high levels in more mature thymocytes that already express the T-cell antigen receptor complex. Immature thymocytes, all of which express p56 $6^{\text {lck }}$, do not express p59 $9^{f y n}$ at any significant level. Careful study revealed that $\mathrm{p} 59^{f y n}$ protein levels increase about 20 -fold at the time when thymocytes acquire the ability to transmit signals from the $\mathrm{T}$-cell antigen receptor and to undergo repertoire selection. For this reason, we sought to perturb the expression of $\mathrm{p} 59^{/ y n}$ and thereby hoped to influence thymocyte signaling properties.

To perform this experiment, we used a version of the lck promoter vector that contains the entire hGH gene (Fig. 5). The presence of this normal gene context greatly improves expression in transgenic animals by a still obscure mechanism. We introduced fyn cDNA into the first exon of the hGH gene. Using this kind of construct, high levels of expression of fyn transcripts were 


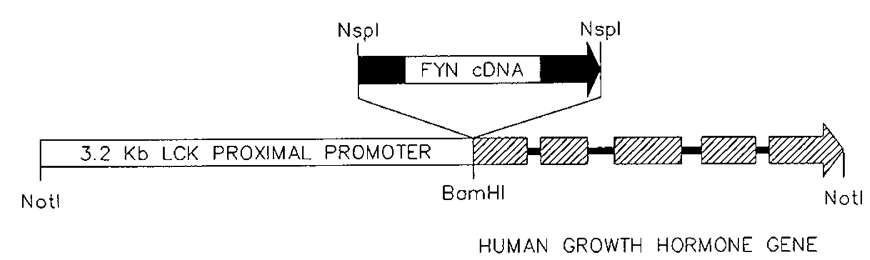

Fig. 5. Structure of a vector that directs expression of $\mathrm{p} 59^{\text {fyn }}$ in transgenic mice. The fyn cDNA is positioned within the first exon of an hGH gene cassette that is itself under the control of the proximal $l c k$ promoter. Adapted from reference 23 .

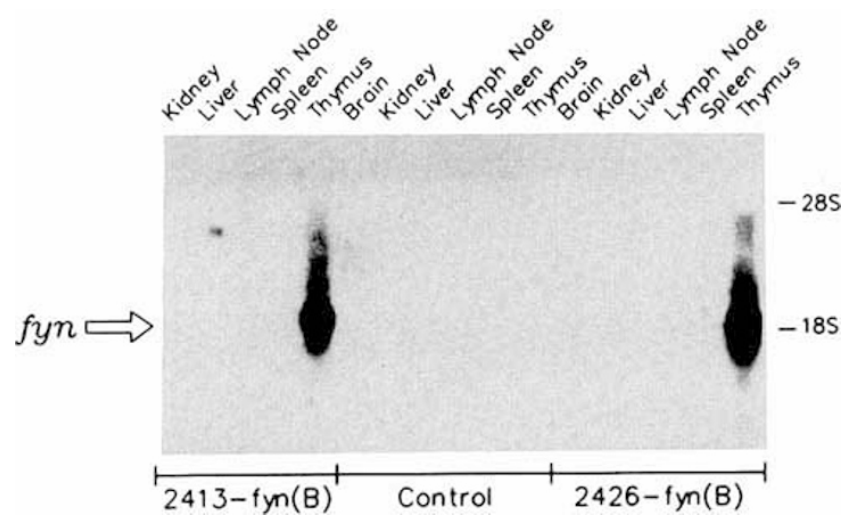

Fig. 6. High-level expression of fyn transcripts in lck-fyn mice. Shown is an RNA blot probed with radiolabeled fyn sequences documenting expression of the fyn transgene in the thymocytes of two different transgenic mice. Adapted from reference 23.

observed in the thymocytes of transgenic animals (Fig. 6) as compared with littermate controls (23). Virtually every transgenic thymocyte expresses $\mathrm{p} 59^{f y n}$ from the proximal $l c k$ promoter; the $559^{f y n}$ is therefore much more abundant and much easier to detect. Of course, no increase in $\mathrm{p} 59^{\text {fyn }}$ expression occurs in the spleen because the proximal lck promoter turns off at the time that thymocytes populate the periphery. Lymph node $T$ cells are similarly unaffected.

Thymocytes that overexpress $\mathrm{p}^{59^{\mathrm{y} n}}$ become, by all criteria, hyperstimulable. In response to antigen receptor stimulation, these thymocytes rapidly augment intracellular calcium levels, release large amounts of IL-2, and proliferate up to 10 times better than normal. An example of the improved calcium response is shown in Figure 7. Transgenic thymocytes respond to lectin mitogen or to anti-CD3 antibodies by rapidly accumulating high levels of intracellular free calcium. The response outstrips that of littermate controls with respect to both rapidity and extent. These observations provoked a much more interesting experiment. A lysine residue within the catalytic domain of p5 $9^{\text {fyn }}$, by analogy with a similar lysine in the catalytic domain of the cAMP-dependent protein kinase, is crucial for phosphate transfer (23). It was possible, by mutating this lysine residue, to create a catalytically inactive version of $\mathrm{p} 59^{\text {fyn }}$. When the nonfunctional kinase was expressed in transgenic thymocytes, no improvement was observed. Hence, the kinase activity of p59 fyn is required for its positive effects on T-cell stimulation.

However, the "dead" fyn experiment proved even more interesting because the presence of the inactive kinase actually compromised the normal proliferative capacity of thymocytes responding to T-cell receptor-derived signals. Thus, although stimulation provokes hyperproliferative behavior in cells expressing the wild-type fyn kinase (these thymocytes proliferate more than 10 -fold faster than do cells derived from normal littermates), thymocytes that express the dead version of the $f y n$ kinase do not proliferate at all (23). This does not result from some global defect in their ability to undergo replication. Stimulation of all
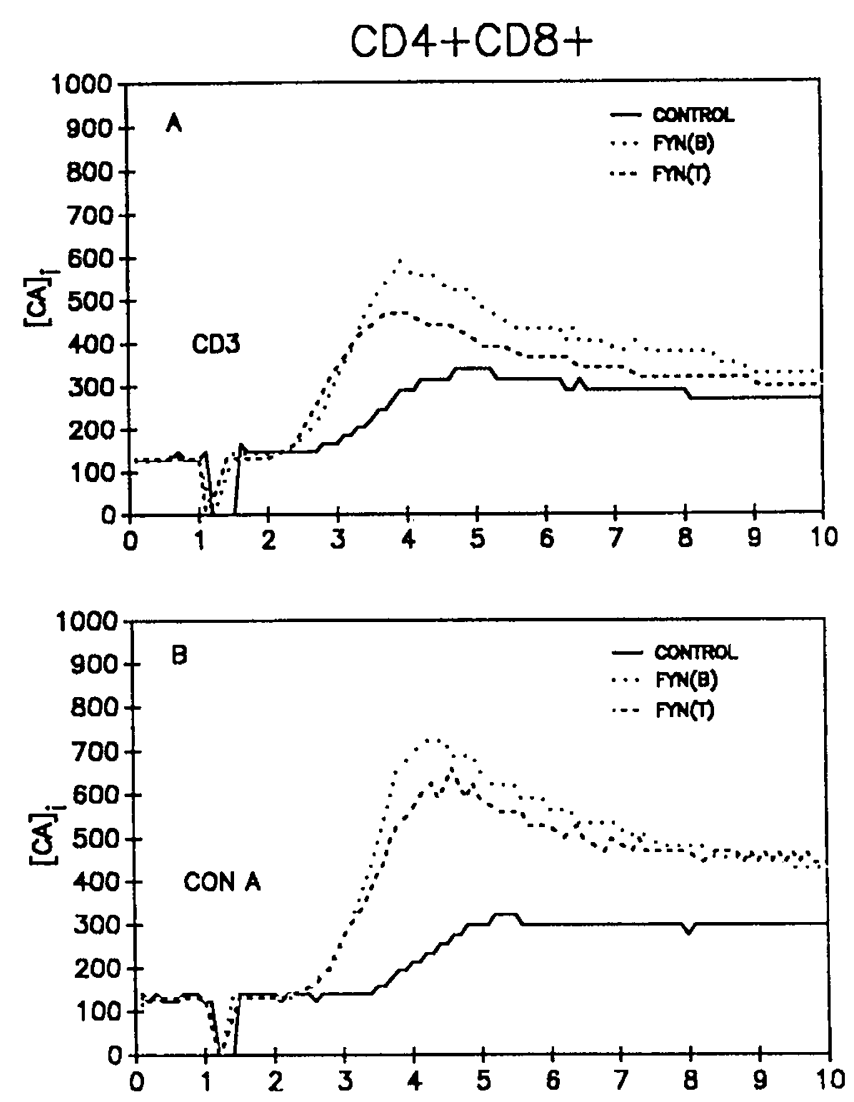

Fig. 7. Improved calcium mobilization in stimulated CD4+8+ thymocytes from lck-fyn transgenic mice. Shown are mean calcium levels in CD4+8+ thymocytes determined using a calcium-sensitive fluorescent dye and time-resolved flow cytometric methods. The dotted lines represent tracings obtained using thymocytes from transgenic animals, and the solid line shows the results obtained when normal thymocytes were used. Stimulation was achieved using antibodies to the $\mathrm{CD} 3$ complex or using a lectin mitogen (Con A, concanavalin A). See reference 23 for details.

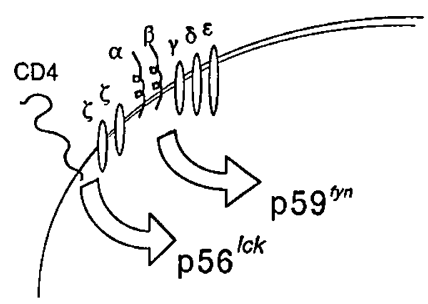

Fig. 8. Two distinct protein tyrosine kinases deliver signals from the T-cell receptor to the cell interior. The structure of the T-cell receptor complex and its signaling components are illustrated schematically.

three types of thymocytes with a calcium ionophore and phorbol ester, thereby bypassing the T-cell receptor, yields identical proliferative responses in each case. Hence, by augmenting the expression of these protein-tyrosine kinases, we have learned something important about the way in which signals are coupled to the T-cell antigen receptor. Antigen recognition by the T-cell receptor no doubt provokes activation of a variety of signal transduction elements. But one of these is certainly $\mathrm{p} 59^{f y n}$, which must be present in active form to permit signaling by mature thymocytes.

Other data permit elaboration of a somewhat improved model for signal transduction from the $\mathrm{T}$-cell antigen receptor complex (Fig. 8). The data reveal that p56 ${ }^{\text {lck }}$ interacts with the CD4 and CD8 coreceptors, p59 $9^{f y n}$ interacts with the T-cell receptor itself 
via its CD3 components, and both of these participate in signal transduction events that mediate lymphocyte activation. These molecules represent potentially important targets for pharmacologic intervention in neoplastic and autoimmune diseases.

Acknowledgments. The author thanks Kathy Forbush, who generated all the transgenic animals used in these experiments, and the very gifted graduate students and postdoctoral fellows who have been involved in performing these studies.

\section{REFERENCES}

1. Ullman KS, Northrop JP, Verweij CL, Crabtree GR 1990 Transmission of signals from the $T$ lymphocyte antigen receptor to the genes responsible for cell proliferation and immune function: the missing link. Annu Rev Immunol 8:421-452

2. Jorgensen JL, Reay PA, Ehrich EW, Davis MM 1992 Molecular components of T-cell recognition. Annu Rev Immunol 10:835-873

3. Minami Y, Weissman AM, Samelson LE, Klausner RD 1988 Building a multichain receptor: synthesis, degradation, and assembly of the T-cell antigen receptor. Proc Natl Acad Sci USA 141:42-49

4. Janeway CA 1992 The $T$ cell receptor as a multicomponent signaling machine: CD4/CD8 coreceptors and CD45 in T cell activation. Annu Rev Immunol 10:645-674

5. Ullrich A, Schlessinger J 1990 Signal transduction by receptors with tyrosine kinase activity. Cell 61:203-212

6. Marth JD, Peet R, Krebs EG, Perlmutter RM 1985 A lymphocyte-specific protein-tyrosine kinase is rearranged and overexpressed in the murine $\mathrm{T}$ cell lymphoma LSTRA. Cell 43:393-404

7. Perlmutter RM, Marth JD, Ziegler SF, Garvin AM, Pawar S, Cooke MP, Abraham KM 1988 Specialized protein tyrosine kinase proto-oncogenes in hematopoietic cells. Biochem Biophys Acta 948:245-262

8. Cooper JA 1989 The $s r c$ family of protein tyrosine kinases. In: Kemp B, Alewood PF (eds) Peptides and Protein Phosphorylation. CRC Press, Boca Raton, FL, pp 85-113

9. Dymecki SM, Niederhuber JE, Desiderio SV 1990 Specific expression of a tyrosine kinase gene, $b l k$, in B lymphoid cells. Science 247:332-336

10. Ziegler SF, Marth JD, Lewis DB, Perlmutter RM 1989 Novel protein-tyrosine kinase gene $(h c k)$ preferentially expressed in cells of hematopoietic origin. Mol Cell Biol 9:2724-2727

11. Marth JD, Cooper JA, King CS, Ziegler SF, Tinker DA, Overell RW, Krebs EG, Perlmutter RM 1988 Neoplastic transformation induced by an activated lymphocyte-specific protein tyrosine kinase $\left(\mathrm{p} 56^{k k}\right)$. Mol Cell Biol 8:540550

12. Marth JD, Disteche C, Pravtcheva D, Ruddle F, Krebs EG, Perlmutter RM 1986 Localization of a lymphocyte-specific protein tyrosine kinase gene (lck) at a site of frequent chromosomal abnormalities in human lymphomas. Proc Natl Acad Sci USA 83:7400-7404

13. Tycko B, Smith SD, Sklar J 1991 Chromosomal translocations joining LCK and TCRB loci in human T cell leukemia. J Exp Med 174:867-873

14. Turner JM, Brodsky MH, Irving BA, Levin SD, Perlmutter RM, Littman DR 1990 Interaction of the unique N-terminal region of the tyrosine kinase p5 $56^{\text {lck }}$ with the cytoplasmic domains of CD4 and CD8 is mediated by cysteine motifs. Cell 60:755-765

15. Mustelin T, Coggeshall KM, Altman A 1989 Rapid activation of the T cell tyrosine protein kinase $\mathrm{p} 56^{\text {lck }}$ by the $\mathrm{CD} 45$ phosphotyrosine phosphatase. Proc Natl Acad Sci USA 86:6302-6306

16. Wildin RS, Garvin AM, Pawar S, Lewis DB, Abraham KM, Forbush KA, Ziegler SF, Allen JM, Perlmutter RM 1991 Developmental regulation of lck gene expression in T lymphocytes. J Exp Med 173:383-393

17. Reynolds PJ, Lesley J, Trotter J, Schulte R, Hyman R, Sefton BM 1990 Changes in the relative abundance of type I and type II lck mRNA transcripts suggest differential promoter usage during T-cell development. Mol Cell Biol $10: 4266-4270$

18. Abraham KM, Levin SD, Marth JD, Forbush KA, Perlmutter RM 1991 Thymic tumorigenesis induced by overexpression of p $56^{\text {lck. }}$. Proc Natl Acad Sci USA 88:3977-3981

19. Hatakeyama M, Kono T, Kobayashi N, Kawahara A, Levin SD, Perlmutter RM, Tanaguchi T 1991 Interaction of the IL-2 receptor with the $s r c$ family kinase p56 $6^{\text {kck }}$ identification of novel intermolecular association. Science 252:1523-1528

20. Takeshita T, Asao H, Ohtani K, Ishii N, Kumaki S, Tanaka N, Munakata H, Mankamura M, Sugamura K 1992 Cloning of the chain of the human IL-2 receptor. Science 257:379-382

21. Cooke MP, Perlmutter RM 1989 Expression of a novel form of the fyn protooncogene in hematopoietic cells. New Biol 1:66-74

22. Appleby MW, Gross JA, Cooke MP, Levin SD, Qian X, Perlmutter RM 1992 Defective $\mathrm{T}$ cell receptor signaling in mice lacking the thymic isoform of p59 $9^{/ y n}$. Cell 70:751-764
23. Cooke MP, Abraham KM, Forbush KA, Perlmutter RM 1991 Regulation of $\mathrm{T}$ cell receptor signaling by a $s r c$ family protein-tyrosine kinase $\left(\mathrm{p} 59^{/ y n}\right)$. Cell $65: 281-291$

\section{FLOOR DISCUSSION}

Dr. Gupta: I have two questions. First, is there a differential regulation of lck and fyn by phorbol esters? Second, we know that if you expose a cell for a prolonged period to phorbol esters and then come back with the normal stimulating T-cell receptor, it does not respond. What has happened to the lck?

Dr. Perlmutter: That is an interesting phenomenon. The $l c k$ encoded protein, $\mathrm{p} 56$, undergoes a modification after activation of T lymphocytes that results in its conversion to forms generically called $\mathrm{p} 60^{\text {lck }}$ that have reduced mobility when visualized using electrophoretic procedures. This modification, associated with serine phosphorylation in the amino-terminal region, can be effectively stimulated by phorbol esters. In the normal activation sequence, virtually all the p56 is converted to this p 60 form, and transcription of the lck gene declines transiently. We think this means that the cell is undergoing some sort of feedback desensitization; functional lck protein disappears, and whatever protein is there is rapidly converted to this p 60 form.

We have been unable to describe precisely what catalytic differences exist between the $p 60$ and the $p 56$ forms. The ability to support this transformation from p56 to p60 is a unique property of hematopoietic cells. If we place p 56 into fibroblasts, for example, phorbol esters will not stimulate that transition from $\mathrm{p} 56$ to $\mathrm{p} 60$. A specific pathway is probably involved in altering the activation sequence.

Dr. Schreiber: Have you looked at serine and threonine phosphorylation in terms of T-cell receptor activation and its potential function?

Dr. Perlmutter: There are a large number of serine/threonine kinases. Numerous investigators have looked at alterations in the activity of certain specific serine/threonine kinases, particularly casein kinase II, the S6 ribosomal kinases, and the map-2 or erk kinases. The activity of these kinases does change during the activation sequence. We suspect that the increase in activity of many of these kinases is the result of this tyrosine phosphorylation-induced cascade. But neither we nor to my knowledge anyone else has evidence that tyrosine phosphorylation directly alters the activity of serine kinases in $\mathrm{T}$ cells. The serine kinases are presumably regulated by some other process. We have not been able to dissect out these steps. It is an area of active investigation.

Dr. Jordan: Current immunosuppressive agents, such as cyclosporine or FK506, are most effective in inhibiting T-cell receptoractivated cells. Is there evidence that they may inhibit tyrosine kinase activity?

Dr. Perlmutter: No, they seem to act later. The tyrosine kinase activation process occurs in cyclosporine-inhibited cells. There is good evidence that cyclosporine and FK506 inhibit events downstream, notably the assembly of the nuclear factor of activated $T$ cells (NFAT) in the nucleus.

Dr. Strober: As you know, there are frequent situations where cell membrane proteins interact with one another. Do you believe the reason for those interactions is to bind some of these kinases better? Is there any evidence for that in the case of $l c k$ or $f y n$ ?

Dr. Perlmutter: Your interesting question brings up a general technical point. Coimmunoprecipitation experiments have become popular in recent years. The experiment simply takes an antibody with which we can, for example, precipitate a cell surface molecule and then tries to make the immunoprecipitate as dirty as possible. The theory is that anything that comes down must somehow be associated physically. These experiments are dangerous. In performing coimmunoprecipitation experiments, at the limit, everything coimmunoprecipitates. The critical question is Can we provide genetic data to show that two molecules are associated and define a specific region of a molecule required 
for the interaction? In many cases, it has been possible to do that. This is a roundabout way of addressing your question.

In certain circumstances, it is apparent that the association of a kinase with a protein-p56, CD4, and CD8 are good examples-is important. We can perform mutations that disrupt that association, leave the kinase intact, and show that these have specific effects on a CD4-mediated process. We have learned from elegant studies done by Singer and Kupfer, Janeway, and others that CD4 associates with the T-cell antigen receptor complex. Thus, there is reason to believe that in such a situation we bring kinases into a complex. Moreover, there is overwhelming evidence from the growth factor-receptor field that proteintyrosine kinases usually are activated by forming multimers. The best hypothesis with regard to kinase regulation is that in one fashion or another we bring more of these molecules together, presumably by bringing cell surface structures together, because, of course, the kinases do not penetrate the membrane. In general, the logic is $100 \%$ correct; the specifics are mysterious.

Dr. Hershfield: Regarding the expression of $l c k$, is that in cells that are absolutely committed to the $T$-cell lineage? If you were to inhibit or in some way block that expression, are the cells in which $l c k$ would have been expressed capable of any other lineage differentiation at that point?

Dr. Perlmutter: I can tell you the results of experiments that address that point. The lck gene is expressed in lymphoid cells and NK (natural killer) cells. In our experience, the gene is expressed in B lymphocytes at levels about 20 -fold lower than in $\mathrm{T}$ cells. The results vary from investigator to investigator. B-cell lines often express p56, but we do not know whether normal B cells really express p 56 or whether there are always some contaminating $\mathrm{T}$ cells or NK cells. Eliminating $l c k$ function has a deleterious effect on T-cell formation. We have shown that effect using the dominant negative approach. Tak Mak has done the same by knocking out the gene.

In either case, the animals do not really have a thymus. They have thymic stroma, but there are only about a million cells in the thymus. The cells are all immature thymoblasts that cannot progress. The animals do have B lymphocytes. It appears from these experiments that $l c k$ is required for thymocyte development, but apparently not for B lymphocyte development, at least in this system.

Dr. Gelfand: In the overexpressed p56 with a predominance of the double negatives, it is thought that some of these cells may express IL-2 receptors that, as you suggest, may be link signaling to $\mathrm{p} 56$. Do these cells respond to IL-2?

Dr. Perlmutter: That is a good question. We have done two relevant experiments. There is a block in development in animals that overexpress p56. We have dissected that block carefully and found it to be remarkably specific. It is a block in the joining of variable regions of the $\beta$ chain to the functioning of DJ joint segments. We correct that block in development simply by putting in a functionally rearranged $T$-cell receptor $\beta$ chain that seems to be independent of IL-2 receptor function. However, in animals that are expressing a dead version of $l c k$, the cells that accumulate are all IL-2 receptor-positive. That is consistent with a model in which $l c k$ is required for an early intrathymic signal, and we have blocked the ability to deliver that signal. But in animals that lack IL-2, thymocyte development proceeds normally. If there is a signal, it is delivered by some other ligand.

Dr. Gelfand: My second question concerns the cause and effect of the kinase, particularly in the $f y n$ overexpression. With the dead kinase, is it possible that you are driving a different cell to differentiate? Is it not so much cause and effect, but rather the ability of the cell to respond?

Dr. Perlmutter: Yes, that is always possible. The cell would have to be common and one that we cannot distinguish using any available markers. We have disrupted the fyn gene in other experiments, so we have animals that do not express this protein. With our method, we disrupt just the exon required for expression of fyn in T cells. They have the fyn gene, but not the specific form. In those animals, the thymocytes do not signal. On stimulating them with anti-T-cell receptor, Con A (concanavalin A), or whatever, we get very little calcium flux, no proliferation, no IL-2 release, and so forth. Despite the fact that the thymocyte number and subset representation are normal, cells leave the thymus and populate the periphery in a normal fashion. The kinetics of this process appear normal. Although signaling is completely disrupted in the ways we usually measure it, that signal is not required for normal development or for normal antigen-specific selection. This intriguing observation suggests that we are probably not recruiting a separate population; we have only eliminated a nonessential function.

Dr. Gelfand: Is this contrary to the CD3-mediated apoptosis hypothesis?

Dr. Perlmutter: Probably not. Other signals probably suffice for CD3-mediated deletion. I believe the apoptosis phenomenon is calcium dependent. In parallel experiments placing the muscarinic receptor into these animals and stimulating them with carbachol, the cells undergo apoptosis beautifully.

Dr. Gelfand: What do you mean by "hyperproliferation"more cells capable of responding, more cycles? Theoretically, the process is an all or nothing phenomenon for a single cell.

Dr. Perlmutter: More cells are capable of responding. Ordinarily, there is a transition point as cells go from double positive to single positive where they up-regulate $\mathrm{p} 59^{f y n}$. We have given $\mathrm{p} 59^{f y n}$ to everyone so that the end result is a lot more proliferation; many more cells effectively couple from the T-cell receptor. About half of the double-positive cells have receptors.

Dr. Stiehm: What would you expect the expression of $l c k$ to be on the $T$ cells of a patient with thymic aplasia-DiGeorge syndrome? Have you had a chance to look?

Dr. Perlmutter: We have not had that chance. What I think is lacking is an appropriate stromal environment for T-cell development. There will of course be relatively few $\mathrm{T}$-lineage precursors stuck there that one can identify. If they have reached the point where $l c k$ is ordinarily expressed - probably about the time they arrive in the thymus-we would still see $l c k$ transcripts. But they would not be able to function because some cue from the stromal environment is missing.

Dr. Levinson: You have discussed the consequences of overexpression of p59 in your transgenic model, at least with respect to hyperproliferation. Do you have any idea of the consequences of overexpression of the $\mathrm{p} 59$ wild-type for the selection of the Tcell repertoire?

Dr. Perlmutter: We have a good idea. That overexpression seems to have almost no consequences. We expected that we would change thymocyte selection dramatically in such populations and see a range of different $\mathrm{T}$-cell receptor types coming out. Every attempt we have made to identify that change has failed. That probably tells us something, but it might be difficult to see a change in T-cell receptor repertoire. Presumably we have altered the threshold for selection. Cells that would ordinarily not be selected at all, and hence would disappear, now can be selected. Cells that ordinarily would be selected now would be negatively selected because they stimulate too well. Such a change might not alter the representation of T-cell receptor $\alpha$ and $\beta$ chain use in any convincing way. If we cross those animals with T-cell receptor transgenics, as we have done, we might not be able to alter the repertoire, because the T-cell receptors used in such experiments are efficient at selection anyway. We cannot make the selection better.

Dr. Levinson: It might not have an effect on the selection of specific $\mathrm{V}$ betas detectable with serologic reagents, but perhaps it could let cells sneak through that otherwise would not have gotten through with regard to autoimmune activity. Have any of those animals survived long enough to take a look at that?

Dr. Perlmutter: Yes, our animals seem to have a normal lifespan. They never develop autoimmune disease that we can detect. Moreover, there are no abnormalities in mixed lympho- 
cyte reactions or in any of those simple things. We had hoped to see that, but did not.

Dr. Ochs: You mentioned several experiments knocking out the $5^{59^{f y n}}$ or inserting a killed $\mathrm{p} 59^{f y n}$. I take it these experiments were done on transgenic mice, and you said they expressed a normal range of lymphocytes in the periphery. Are they actually functioning? Are these animals "normal immune" and do they respond to antigens?
Dr. Perlmutter: The best way to address that question relates to the fyn knockout animal group, the situation where we do not have the gene. Frankly, we do not have enough data to say. From a biochemical standpoint, there are subtle abnormalities in $\mathrm{T}$ cell stimulation. Normal numbers of cells exist in the periphery, but we do not have enough data on real antigen responses to provide a comprehensive answer on functioning. But soon we will! 